\title{
Outcomes for Relapsed and Refractory Peripheral T-Cell Lymphoma Patients after Front-Line Therapy from the COMPLETE Registry
}

\author{
Frederick Lansigan $^{a}$ Steven M. Horwitz ${ }^{b}$ Lauren C. Pinter-Brown ${ }^{c}$ Steven T. Rosen ${ }^{d}$ \\ Barbara Pro $^{\text {e Eric D. Hsi }}{ }^{f}$ Massimo Federicog ${ }^{9}$ Christian Gisselbrecht $^{\text {h }}$
} Marc Schwartzi Lisa A. Bellm ${ }^{j} \quad$ Mark Acostak $^{k}$ Andrei R. Shustov' Ranjana H. Advanim ${ }^{m}$ Tatyana Feldman $^{n}$ Mary Jo Lechowicz ${ }^{\circ}$ Sonali M. Smith ${ }^{p}$ Anil Tulpule ${ }^{q}$ Michael D. Craig ${ }^{r} \quad$ John P. Greer ${ }^{\mathrm{s}} \quad$ Brad S. Kahl $^{\mathrm{t}} \quad$ Joseph W. Leach ${ }^{\mathrm{u}}$ Neil Morganstein ${ }^{v}$ Carla Casulow ${ }^{\mathrm{w}}$ Steven I. Park ${ }^{\mathrm{x}}$ Francine M. Foss ${ }^{\mathrm{y}}$

\begin{abstract}
${ }^{a}$ Dartmouth Hitchcock Medical Center, Hanover, NH, USA; ${ }^{b}$ Memorial Sloan-Kettering Cancer Center, New York, NY, USA; ' University of California, Irvine, Irvine, CA, USA; ${ }^{d}$ City of Hope, Duarte, CA, USA; ${ }^{e}$ Robert H. Lurie Comprehensive Cancer Center, Northwestern University, Chicago, IL, USA; ${ }^{\mathrm{C} C l e v e l a n d} \mathrm{Clinic}, \mathrm{Cleveland}, \mathrm{OH}, \mathrm{USA} ;{ }^{9}$ Centro Oncologico Modenese, Policlinico, Modena, Italy; ${ }^{\text {h}}$ Hôpital Saint Louis, Paris, France; i MS Biostatistics, LLC, Clermont, FL, USA; ${ }^{j}$ MedNet Solutions, Minnetonka, MN, USA; ${ }^{k}$ Spectrum Pharmaceuticals Inc., Irvine, CA, USA; 'Fred Hutchinson Cancer Research Center, Seattle, WA, USA; ${ }^{m}$ Stanford University Medical Center, Stanford, CA, USA; ${ }^{n}$ Hackensack University Medical Center, Hackensack, NJ, USA; ${ }^{\circ}$ Emory University, Atlanta, GA, USA; ${ }^{P}$ University of Chicago, Chicago, IL, USA; ${ }^{9}$ University of Southern California, Los Angeles, CA, USA; r'West Virginia University, Morgantown, WV, USA; ${ }^{5}$ Vanderbilt University Medical Center, Nashville, TN, USA; ${ }^{t}$ Washington University School of Medicine, St. Louis, MO, USA; "Virginia Piper Cancer Institute, Minneapolis, MN, USA; ${ }^{\vee}$ Overlook Medical Center, Summit, NJ, USA; ${ }^{w}$ University of Rochester, Rochester, NY, USA; ${ }^{x}$ Levine Cancer Institute, Chapel Hill, NC, USA; 'y Yale University, New Haven, CT, USA
\end{abstract}

\section{Keywords \\ Prospective cohort studies - Peripheral T-cell lymphoma • Drug therapy · Combination · Salvage therapy}

\begin{abstract}
Background: Outcomes for patients with peripheral T-cell lymphoma (PTCL) who fail to achieve complete response $(\mathrm{CR})$ or relapse after front-line therapy are poor with lack of prospective outcomes data. Objectives: COMPLETE is a prospective registry of 499 patients enrolled at academic and community sites in the United States detailing patient demographics, treatment and outcomes for patients with ag-
\end{abstract}

gressive T cell lymphomas. We report results for patients with primary refractory and relapsed disease. Methods: Primary refractory disease was defined as an evaluable best response to initial treatment (induction \pm maintenance or consolidation/transplant) other than $\mathrm{CR}$, and included a partial response, progressive disease, or no response/stable disease. Relapsed disease was defined as an evaluable best response to initial treatment of $C R$, followed by disease progression at a later date, irrespective of time to progression. Patients were included in the analysis if initial treatment be-

Presented in Part at the American Society of Hematology (ASH) 59th Annual Meeting, December 9-12, 2017, San Francisco, CA, USA.

\section{KARGER}

(c) 2019 S. Karger AG, Basel

E-Mail karger@karger.com

www.karger.com/aha
Dr. Francine M. Foss

Yale University School of Medicine

333 Cedar Street

New Haven, CT 06510 (USA)

E-Mail Francine.foss@yale.edu 
gan within 30 days of enrollment and treatment duration was $\geq 4$ days. Results: Of 420 evaluable patients, 97 met the definition for primary refractory and 58 with relapsed disease. In the second-line setting, relapsed patients received single-agent therapies more often than refractory patients (52 vs. $28 \% ; p=0.01$ ) and were more likely to receive singleagent regimens ( 74 vs. $53 \% ; p=0.03$ ). The objective response rate to second-line therapy was higher in relapsed patients (61 vs. $40 \% ; p=0.04$ ) as was the proportion achieving a CR (41 vs. $14 \% ; p=0.002$ ). Further, relapsed patients had longer overall survival (OS) compared to refractory patients, with a median OS of 29.1 versus 12.3 months. Conclusions: Despite the availability of newer active single agents, refractory patients were less likely to receive these therapies and continue to have inferior outcomes compared to those with relapsed disease. PTCL in the real world remains an unmet medical need, and improvements in front-line therapies are needed.

(c) 2019 S. Karger AG, Basel

\section{Introduction}

Peripheral T-cell lymphomas (PTCL) encompass a biologically and clinically diverse group of rare and usually aggressive diseases arising from mature $\mathrm{T}$ and natural killer (NK) cells. Despite their heterogeneous pathologic appearance and clinical presentations, the most common entities, PTCL-not otherwise specified (PTCL-NOS), angioimmunoblastic T-cell lymphoma, and anaplastic large cell lymphoma (ALCL), are typically treated similarly with standard CHOP-based (cyclophosphamide, doxorubicin, vincristine and prednisone) regimens followed by autologous stem cell transplantation (ASCT) in suitable patients $[1,2]$. The prognosis for patients with PTCL, from historical retrospective studies, is generally poor [3], with a treatment course characterized by multiple relapses and/or initial refractory disease [4].

According to the NCCN guidelines, a number of treatment strategies can be employed for patients with relapsed or refractory disease, including multi-agent chemotherapy and single-agent therapies, including FDAapproved agents such as pralatrexate (2009), romidepsin (2011), brentuximab vedotin (2011, systemic ALCL only), and belinostat (2014) [5-7]. The role of ASCT in the relapsed and refractory setting is evolving, with some data suggesting that allogeneic transplantation may be better for patients with resistant or relapsed disease [8-10]. The treatment decisions for patients with relapsed or refractory disease are confounded by patient factors such as co- morbidities as well as lack of randomized clinical trials informing the selection of strategies or agents for specific PTCL subtypes [2].

To examine the real-world outcomes for patients who progress after front-line therapy or have primary refractory disease, we analyzed data from the COMPLETE registry, a prospective multicenter cohort study of the demographics, treatment, and outcomes for patients with aggressive $\mathrm{T}$ cell lymphomas from 56 centers representing both academic and community sites within the United States (NCT01110733). For this analysis, we defined a population of patients who did not achieve a complete response (CR) to front-line therapy as determined by the treating physician and compared those who relapsed after CR with those who were refractory to front-line therapy. We report outcomes by disease subtype and treatment strategies.

\section{Material and Methods}

\section{Study Eligibility}

Patients in COMPLETE were enrolled at first diagnosis of histologically confirmed PTCL, after providing written informed consent, and were followed prospectively for subsequent treatment(s) and outcomes. All subtypes of T-cell lymphoma were allowed in the study except for those considered more indolent: T-cell large granular lymphocytic leukemia, primary cutaneous CD30+ disorders, and mycosis fungoides (MF) other than transformed MF, and Sézary syndrome. Patients were enrolled from practices that were self-designated as community or academic. Concomitant participation in clinical trials was permitted.

\section{Cohort Selection}

The dataset analyzed in this study was defined based on study entry criteria. Patients were allowed to enter the study if they signed consent within 30 days after first therapy. The study allowed for patients with acute onset of disease requiring immediate therapy to be enrolled so as to not bias against patients with adverse factors. Patients were excluded from the dataset if they initiated front-line therapy either 30 days prior or after signing informed consent. Due to potentially different outcomes of patients with refractory PTCL after front-line therapy compared to those with initial chemotherapy sensitive disease [11], patients were grouped and analyzed separately. Primary refractory disease was defined in this analysis as an evaluable best response to initial treatment (induction \pm maintenance or consolidation/transplant) as recorded in the database by the treating physician as other than CR, including a partial response (PR), progressive disease (PD), or no response/stable disease (SD). Relapsed disease was defined as an evaluable best response to initial treatment of CR as recorded in the database by the treating physician, followed by disease progression at a later date, irrespective of the time to progression. Patients were included in the analysis if their initial treatment for PTCL began within 30 days of enrollment in the study and treatment duration as recorded in the database was at least 4 days. 
Treatment and clinical responses were determined by the treating investigator. Baseline, initial treatment and follow-up records in the database were required to be approved/locked by the principal investigator for a patient to be included in this analysis.

\section{Statistical Analysis}

Descriptive statistics were used to summarize the baseline characteristics of the study cohorts. For categorical and ordinal variables, frequencies and percentages were calculated. For continuous variables, descriptive statistics (number of patients, mean, median, standard deviation and range) were provided. Null hypothesis testing utilized the $\chi^{2}$ test, $t$ test, and other nonparametric tests as required, with a two-tailed $p$ value of $\leq 0.05$ to reject the null hypothesis. Survival-based analyses were performed using Kaplan-Meier methodology with right censoring as appropriate and evaluated using a log-rank test with a two-tailed $p$ value of $\leq 0.05$ to reject the null hypothesis. For all time-to-event analyses, date of informed consent was used as time zero. All analyses were performed using R version 3.1.0 or greater (The R Foundation for Statistical Computing; https://www.r-project.org/).

\section{Results}

A total of 499 PTCL patients were enrolled in the COMPLETE registry between February 2010 and February 2014 from 41 academic- and 15 community-based practices. Eighteen patients discontinued the study before initial treatment for reasons other than death (eligibility violation, $n=8$; patient lost to follow-up, $n=7$; patient withdrew consent, $n=1$, and site reasons, $n=2$ ). Of the remaining patients, 420 had locked baseline, initial treatment, and follow-up records required for the analysis; overall $54 \%$ of patients $(n=227)$ had a CR to initial therapy. Of the 151 primary refractory patients identified based upon initial treatment response requirements (PR, $\mathrm{PD}$, or $\mathrm{SD}$ ), 54 were censored due to prolonged treatment/informed consent intervals as defined above (informed consent signed $>30$ days from start of treatment), and initial treatment durations either missing or being $\leq 4$ days from signed consent, leaving 97 patients available for evaluation. Similarly, of the 68 patients that met the relapse disease criteria for initial treatment response (CR) followed by a progression, 10 were censored due to similar considerations as above, leaving 58 patients for evaluation.

Characteristics of the patient populations in each group are shown in Table 1 and are representative of PTCL patients reported by the Surveillance, Epidemiology, and End Results (SEER) Program and the International T-Cell Project [12]. The median ages for refractory and relapse groups were 62 and 59, respectively. A high percentage (30\%) of patients in the combined population (refractory and relapsed) had extranodal types of
T cell lymphoma (NK T cell, transformed MF, hepatosplenic $\mathrm{T}$ cell lymphoma, adult $\mathrm{T}$ cell leukemia/lympho$\mathrm{ma}$ ), suggesting that front-line therapies have a low cure rate for these patients. The median follow-up for the refractory cohort was 701 days (interquartile range [IQR] 269-1,108 days), and for the relapsed cohort was 777 days (IQR 462-1,333 days). The median time from diagnosis to determination of primary refractory disease was 3.8 months (IQR 2.4-6). The median time from diagnosis to designation of relapsed disease for the relapsed group was 12.1 months (IQR 8.5-20).

\section{Treatment of Relapsed versus Refractory PTCL Patients}

The treatment approach for all patients was determined by the treating physicians. The COMPLETE registry did not query treating physicians on factors implicated in their treatment choices for relapsed or refractory patients but did query physicians on the goal of secondline therapy (cure vs. palliation). Cure was cited as the goal in the majority of patients (66\% of relapse patients and $59 \%$ of refractory patients). The remaining patients were designated to be treated with palliative intent. To analyze objective patient characteristics potentially influencing the decision regarding curative versus palliative intent in relapsed or refractory patient groups, a multivariable regression model was performed using age (as a continuous variable), ECOG performance status, treatment regimen (single- vs. multi-agent), and treatment status (refractory vs. relapse) as factors. The only factor which was associated with noncurative intent decision was age (odds ratio 1.1, 95\% confidence interval [CI] $1.03-1.12 ; p=0.002)$; other variables were not significantly different between the two groups.

Treatment details for patients receiving systemic therapy for refractory or relapse disease are shown in Table 2. Interestingly, combination chemotherapy was more often in refractory patients compared to relapsed patients ( 72 vs. $48 \%, p=0.01$ ). Relapsed patients were more likely to receive single agents ( 74 vs. $53 \%)(p=0.03)$. In the 62 relapsed and refractory patients who received singleagent therapy, the most common agents were brentuximab vedotin (19\%), romidepsin (14\%), pralatrexate $(10 \%)$, and gemcitabine (5\%). In the 35 patients who received combination therapy, the most common multiagent regimens were ICE (ifosfamide, carboplatin, and etoposide) (17\%), GemOx (gemcitabine and oxaliplatin) (17\%), GND (gemcitabine, navelbine, doxorubicin), and other gemcitabine-based regimens (14\%), platinumbased regimens (12\%), and CHOP (cyclophosphamide,
Lansigan et al. 
Table 1. Demographics and clinical characteristics of patients in first relapse compared to patients with primary refractory disease (at initial diagnosis of PTCL)

\begin{tabular}{|c|c|c|c|c|c|}
\hline \multirow[t]{2}{*}{ Characteristic } & \multicolumn{2}{|c|}{$\begin{array}{l}\text { First relapse } \\
(n=58)\end{array}$} & \multicolumn{2}{|c|}{$\begin{array}{l}\text { Primary refractory } \\
(n=97)\end{array}$} & \multirow[t]{2}{*}{$p$ value } \\
\hline & $n$ & $\%$ & $n$ & $\%$ & \\
\hline Median age (IQR), years & \multicolumn{2}{|c|}{$69(49-73)$} & \multicolumn{2}{|c|}{$62(48-70)$} & 0.61 \\
\hline Sex, male & 40 & 69 & 61 & 63 & 0.44 \\
\hline \multicolumn{5}{|l|}{ ECOG performance status } & 0.55 \\
\hline $0-1$ & 56 & 97 & 90 & 93 & \\
\hline 2 & 2 & 3 & 6 & 6 & \\
\hline$\geq 3$ & 0 & 0 & 1 & 1 & \\
\hline \multicolumn{5}{|l|}{ IPI score } & 0.67 \\
\hline$<2$ & 27 & 28 & 18 & 31 & \\
\hline$\geq 2$ & 40 & 69 & 70 & 72 & \\
\hline \multicolumn{6}{|l|}{ Histology } \\
\hline PTCL-NOS & 25 & 26 & 25 & 29 & 0.11 \\
\hline AITL & 13 & 22 & 14 & 14 & 0.21 \\
\hline ALCL & 6 & 10 & 14 & 14 & 0.46 \\
\hline ALK negative & 5 & 83 & 10 & 71 & 0.57 \\
\hline ALK positive & 1 & 17 & 4 & 29 & \\
\hline T/NK-cell lymphoma, nasal type & 7 & 12 & 15 & 16 & 0.56 \\
\hline Enteropathy-type intestinal TCL & 2 & 3 & 3 & 3 & 0.90 \\
\hline Hepatosplenic TCL & 1 & 2 & 5 & 5 & 0.28 \\
\hline Adult T-cell leukemia/lymphoma (HTLV 1+) & 1 & 2 & 4 & 4 & 0.41 \\
\hline Transformed mycosis fungoides & 0 & 0 & 9 & 9 & 0.02 \\
\hline Other & 6 & 10 & 7 & 7 & 0.50 \\
\hline \multicolumn{5}{|l|}{ Ann Arbor stage } & 0.65 \\
\hline $\mathrm{I} / \mathrm{II}$ & 15 & 26 & 22 & 23 & \\
\hline III/IV & 43 & 74 & 75 & 77 & \\
\hline Bone marrow involvement ${ }^{\mathrm{a}}$ & 10 & 32 & 18 & 27 & 0.58 \\
\hline Elevated $\mathrm{LDH}^{\mathrm{b}}$ & 26 & 45 & 43 & 46 & 0.91 \\
\hline \multicolumn{5}{|l|}{ Prior treatment approach } & 0.003 \\
\hline Chemotherapy/single agent therapy alone & 35 & 60 & 77 & 79 & \\
\hline Stem cell transplant & 17 & 29 & 8 & 8 & \\
\hline Other & 6 & 10 & 12 & 12 & \\
\hline \multicolumn{5}{|l|}{ Prior regimen } & 0.07 \\
\hline Combination regimen & 46 & 81 & 63 & 67 & \\
\hline Single-agent regimen & 11 & 19 & 31 & 33 & \\
\hline Prior participation in clinical trial for PTCL & 14 & 24 & 17 & 18 & 0.32 \\
\hline \multicolumn{5}{|l|}{ Median time from PTCL diagnosis to relapsed } & \\
\hline \multicolumn{6}{|l|}{ Time to relapse from front-line treatment, months } \\
\hline$<3$ & 7 & 12 & - & - & \\
\hline $3-6$ & 16 & 27 & - & - & \\
\hline$>6-12$ & 18 & 31 & - & - & \\
\hline$>12$ & 17 & 29 & - & - & \\
\hline
\end{tabular}

Sum may exceed $100 \%$ due to rounding. PTCL, peripheral T-cell lymphoma; IQR, interquartile range; ECOG, Eastern Cooperative Oncology Group; IPI, International Prognostic Index; PIT, Prognostic Index for PTCL; PTCL-NOS, PTCL-not otherwise specified; AITL, angioimmunoblastic T-cell lymphoma; ALCL, anaplastic large cell lymphoma; ALK, anaplastic lymphoma kinase; NK, natural killer; TCL, T-cell lymphoma; HTLV 1+, human T-cell leukemia virus type $1 ; \mathrm{LDH}$, lactate dehydrogenase; IQR, interquartile range. ${ }^{a}$ Bone marrow involvement information is missing for 27 relapsed patients and 30 primary refractory patients. ${ }^{b} \mathrm{LDH}$ value is missing for 3 primary refractory patients. 
Table 2. Summary of systemic therapy for PTCL patients in first relapse compared to patients with primary refractory disease

\begin{tabular}{|c|c|c|c|c|c|}
\hline \multirow[t]{2}{*}{ Systemic therapy } & \multicolumn{2}{|c|}{$\begin{array}{l}\text { First relapse } \\
(n=50)\end{array}$} & \multicolumn{2}{|c|}{$\begin{array}{l}\text { Primary refractory } \\
(n=47)\end{array}$} & \multirow[t]{2}{*}{$p$ value } \\
\hline & $n$ & $\%$ & $n$ & $\%$ & \\
\hline Regimens & & & & & 0.03 \\
\hline Single agent & 37 & 74 & 25 & 53 & \\
\hline Multi-agent & 13 & 26 & 22 & 47 & \\
\hline Type of drug therapy & & & & & 0.23 \\
\hline Monoclonal antibodies/immunoconjugates & 11 & 22 & 4 & 9 & \\
\hline Gemcitabine-containing regimens & 6 & 12 & 8 & 17 & \\
\hline HDAC inhibitors & 8 & 16 & 3 & 6 & \\
\hline Antifolates & 5 & 10 & 7 & 15 & \\
\hline Platinum-containing regimens & 7 & 14 & 8 & 17 & \\
\hline $\mathrm{L}$-asparaginase-containing regimens & 1 & 2 & 0 & 0 & \\
\hline Other & 12 & 22 & 17 & 36 & \\
\hline
\end{tabular}

Single-agent therapies include histone deacetylase (HDAC) inhibitors, monoclonal antibodies/ immunoconjugates, targeted therapies, and immunomodulatory drugs.

Table 3. Best overall response to second-line therapy for PTCL patients: comparison of relapsed versus refractory patients and of patients who received single agent versus combination therapy

\begin{tabular}{|c|c|c|c|c|c|c|c|c|c|c|}
\hline \multirow[t]{3}{*}{ Best response } & \multicolumn{5}{|c|}{ Patient cohort } & \multicolumn{5}{|c|}{ Type of systemic therapy } \\
\hline & \multicolumn{2}{|c|}{$\begin{array}{l}\text { first relapse } \\
(n=56)\end{array}$} & \multicolumn{2}{|c|}{$\begin{array}{l}\text { primary } \\
\text { refractory } \\
(n=61)\end{array}$} & \multirow[t]{2}{*}{$p$ value } & \multicolumn{2}{|c|}{$\begin{array}{l}\text { single agent } \\
(n=39)\end{array}$} & \multicolumn{2}{|c|}{$\begin{array}{l}\text { combination } \\
\text { therapy } \\
(n=58)\end{array}$} & \multirow[t]{2}{*}{$p$ value } \\
\hline & $n$ & $\%$ & $n$ & $\%$ & & $n$ & $\%$ & $n$ & $\%$ & \\
\hline \multicolumn{11}{|l|}{ Distribution of response } \\
\hline Complete response (CR) & 21 & 41 & 7 & 14 & & 14 & 38 & 12 & 21 & \\
\hline Partial response $(\mathrm{PR})$ & 10 & 20 & 13 & 26 & & 8 & 22 & 13 & 23 & \\
\hline \multicolumn{11}{|l|}{ Stable disease/no } \\
\hline response & 5 & 10 & 12 & 24 & & 3 & 8 & 13 & 23 & \\
\hline Progressive disease & 13 & 26 & 14 & 28 & & 10 & 27 & 15 & 26 & \\
\hline Not evaluable & 2 & 4 & 4 & 8 & & 2 & 5 & 4 & 7 & \\
\hline \multicolumn{7}{|l|}{ Objective response rate } & 60 & 25 & 44 & 0.14 \\
\hline $\mathrm{CR}$ rate & 21 & 41 & 7 & 14 & 0.002 & 14 & 38 & 12 & 21 & 0.08 \\
\hline
\end{tabular}

Response information is not available for 5 relapsed patients, 11 refractory patients, 2 patients who received single agents, and 1 patient who received traditional therapies.

doxorubicin, vincristine, and prednisone) or CHOP-like plus etoposide (9\%). In the relapsed patients, there was a higher ORR (61\%) compared to refractory patients (41\%) $(p=0.04)$, and a higher rate of CR (41 vs. $14 \%)(p=0.002)$. For the entire cohort, the ORR was higher for single- agent treatment compared to multi-agent chemotherapy, although the difference did not reach significance (60 vs. $44 \%)(p=0.14)$ (Table 3).

Fourteen responders went to stem cell transplantation (SCT) (7 allogeneic, 7 autologous), including 10 pa- 


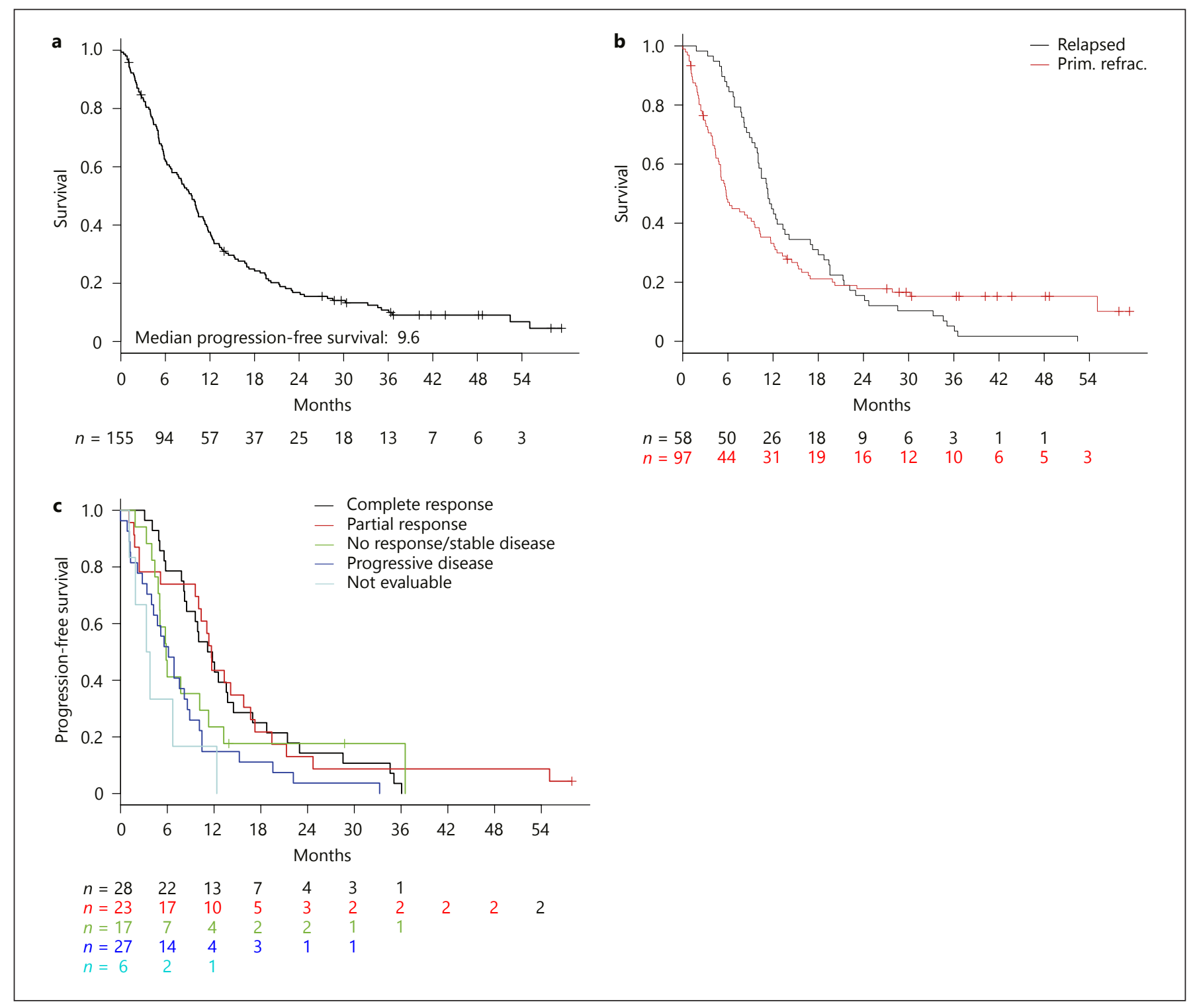

Fig. 1. Kaplan-Meier estimates of progression-free survival of relapsed and refractory PTCL cohorts combined (a), primary refractory versus relapsed patients (b), by response to re-treatment (c).

tients in relapsed and 4 in refractory groups. Of those, $10(71 \%)$ had received single agents and 4 had received multi-agent chemotherapy as a salvage and a bridge to transplant $(p=0.01)$. Altogether, of 155 patients in this analysis (97 refractory, 58 relapsed), only a small proportion $(9 \%)$ had transplant as a part of their salvage therapy.

\section{Survival and Progression-Free Survival}

Median follow-up was similar for relapsed and refractory patients (25.5 months [IQR: 15.2-43.8 months] and 23.0 months [IQR: 8.8-36.4 months], respectively). Kaplan-Meier estimate of median overall survival (OS) was longer for relapsed (29.1 months [95\% CI, 22.1-48.4 months]) compared to refractory patients (12.3 months [95\% CI, 9.1-23.8 months]). One-year survivals were significantly impacted by response to initial therapy with $84 \%$ survival (95\% CI: 76-94\%) for relapsed patients and 52\% (95\% CI: 43-63\%) for refractory patients as shown in Figure 2. Among all relapsed and refractory patients, survival was significantly longer in patients achieving a CR to second-line therapy 


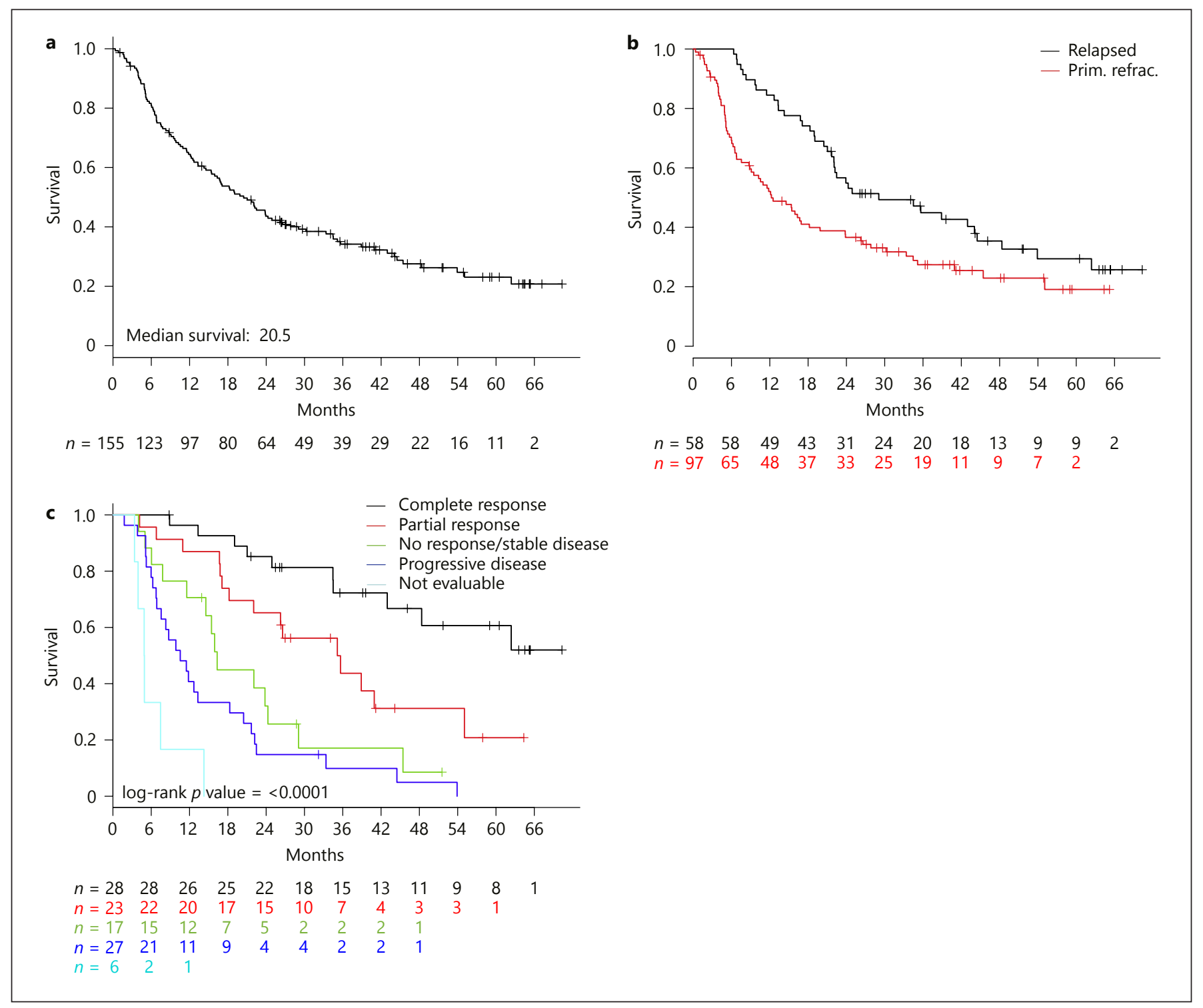

Fig. 2. Kaplan-Meier estimates of overall survival of relapsed and refractory PTCL cohorts combined (a), stratified by relapsed versus refractory disease (b), and stratified by treatment response (c).

compared to those achieving a PR or less $(p=0.0001)$ (Fig. 1c). Median survival was not reached in CR patients (95\% CI, 43 months+) and was 35.2 months (22+) in PR patients as shown in Figure 2. Figure 3 shows outcomes based on single agent vs combination chemotherapy. There was no difference in progression free survival or overall survival based on treatment approach.

Factors influencing OS are shown in Figure 4. The median survival was similar in both groups for ALCL and PTCL-NOS (Fig. 4a, b). As shown in Figure 4c, d, signifi- cant differences were seen in OS by second-line treatment intent, with median survival 43 months (95\% CI: 29.1 months to not reached) for those treated with curative intent compared to those treated with palliative intent: median survival 19 months (95\% CI: 13.3 months to not reached) in relapsed (Fig. 4c) and 15.4 months (95\% CI: 8.9-34.5 months) versus 6.8 months (95\% CI: 4-35.2 months) $(p=0.12)$ in refractory (Fig. $4 \mathrm{~d})$. Differences in outcomes based on single-agent versus combination chemotherapy are shown for each group in Figure $4 \mathrm{e}, \mathrm{f}$. The use of ASCT was associated with a significant increase in 


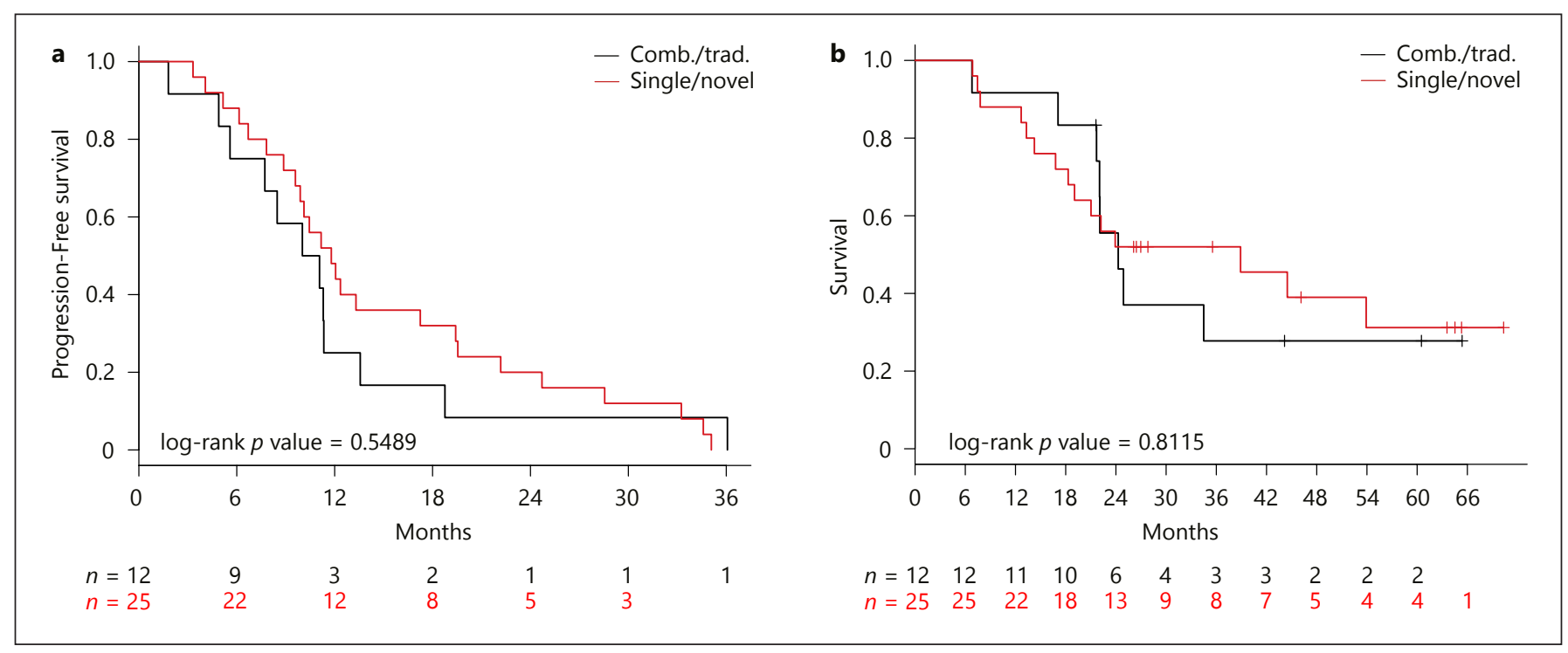

Fig. 3. Kaplan-Meier estimates of progression-free survival of single agent versus combination patients - first retreatment (a) and overall survival of single agent versus combination patients (b).

OS of refractory patients with median survival not reached (95\% CI: 34.5 months to not reached) versus 11.6 months (95\% CI: 6.8-18.2 months) for patients receiving chemotherapy/single-agent therapy (Fig. 4f).

\section{Discussion/Conclusion}

This prospective study of patients enrolled in the COMPLETE registry at initial diagnosis describes realworld patterns of care and outcomes for 155 patients with relapsed or refractory aggressive $\mathrm{T}$ cell lymphomas. While pathology was not reviewed by a reference pathologist, patients who were identified to have poor prognosis histopathology, including adult $\mathrm{T}$ cell leukemia/lymphoma, NK T cell, and other extranodal T cell lymphomas, had a higher chance of having primary refractory disease. With the exception of bone marrow involvement and histopathologic subtype, clinical characteristics of patients at diagnosis were not strongly associated with risk of relapse or refractoriness after front-line therapy. The median survival for relapsed patients was significantly better at 29 months compared to only 12 months for refractory patients. Surprisingly, relapsed patients were more likely to be treated with single agents, while refractory patients were more likely to be treated with combination chemotherapy regimens, despite the fact that refractory patients by definition had demonstrated lack of responsiveness to upfront treatments which were mostly conventional cytotoxic chemotherapy regimens. While our study did not specifically address this issue, it may be that physicians have a bias against the use of single-agent therapies which they perceive may take longer to work in patients with refractory disease.

These data confirm the results of other prospective trials reporting that lack of response to front-line therapy or inherent chemoresistance occurs frequently in patients with aggressive $\mathrm{T}$ cell lymphomas. The prospective Nordic trial of CHOEP followed by SCT documented that only $63 \%$ of patients had a CR to front-line therapy and $18 \%$ had relapsed by 2 years [13]. In our study, most patients received a CHOP-like front-line therapy, and a comparable proportion (54\%) had a CR [11-14]. Given the poor outcomes for patients who do not achieve a CR in the front-line, novel strategies are needed to improve front-line therapy.

The proportion of responding patients receiving an SCT to consolidate their response in the relapsed and refractory setting was low. Unexpectedly, we found that single agents appeared to serve as a bridge to SCT more often than traditional combination regimens, although this observation is confounded by selection bias on the part of the treating physician. Randomized controlled studies are needed to further confirm and define the benefit of SCT in relapsed and refractory PTCL. All future randomized studies should consider stratifying by relapsed versus refractory disease given the important prognostic differences shown in this study. 


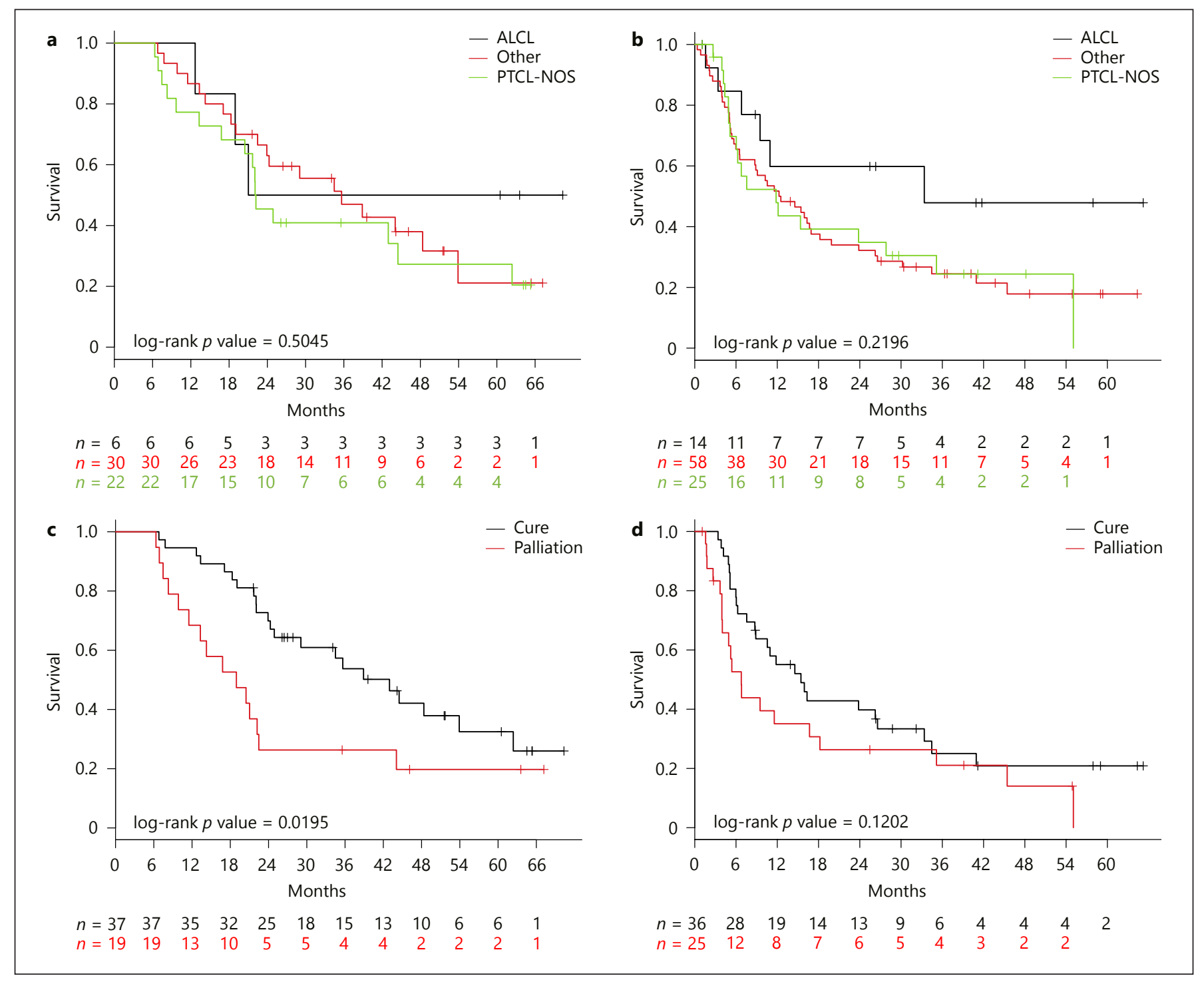

Fig. 4. Kaplan-Meier estimates of overall survival stratified by relapsed PTCL subtype (a), refractory PTCL subtype (b), retreatment intent relapsed (c), retreatment intent refractory $(\mathbf{d})$, type of retreatment, relapsed (e), and type of retreatment, refractory $(\mathbf{f})$.

(Figure continued on next page.)

Our results demonstrated that relapsed patients achieved a better overall response (61 vs. $40 \%$ ) and CR (41 vs. $14 \%)$ to second-line therapy than refractory patients and had a better OS ( 29 vs. 12 months). Our results demonstrate a better survival than reported in a Canadian retrospective study by Mak et al. [4] who reported a median OS of 5.7 months for relapsed and refractory PTCL patients. In this study, 38 patients undergoing ASCT were excluded from the analysis. Of note, half of the patients included in the Mak retrospective database experienced a relapse between 1980 and 2000, well before the era of newer aggressive strategies and supportive care modali- ties and single-agent therapies such as HDAC inhibitors, pralatrexate, and brentuximab vedotin. Additionally, Mak et al. [4] analyzed relapsed and refractory patients together and limited the analysis to the three most common nodal subtypes and excluded patients who were SCT candidates. Our study suggests that SCT in the secondline is feasible and may have an impact on survival in the refractory setting, although there were only 4 patients in the SCT subgroup.

Our results are similar to those recently reported by the T-Cell Project, another similar prospective international T-cell lymphoma registry enrolling PTCL patients 


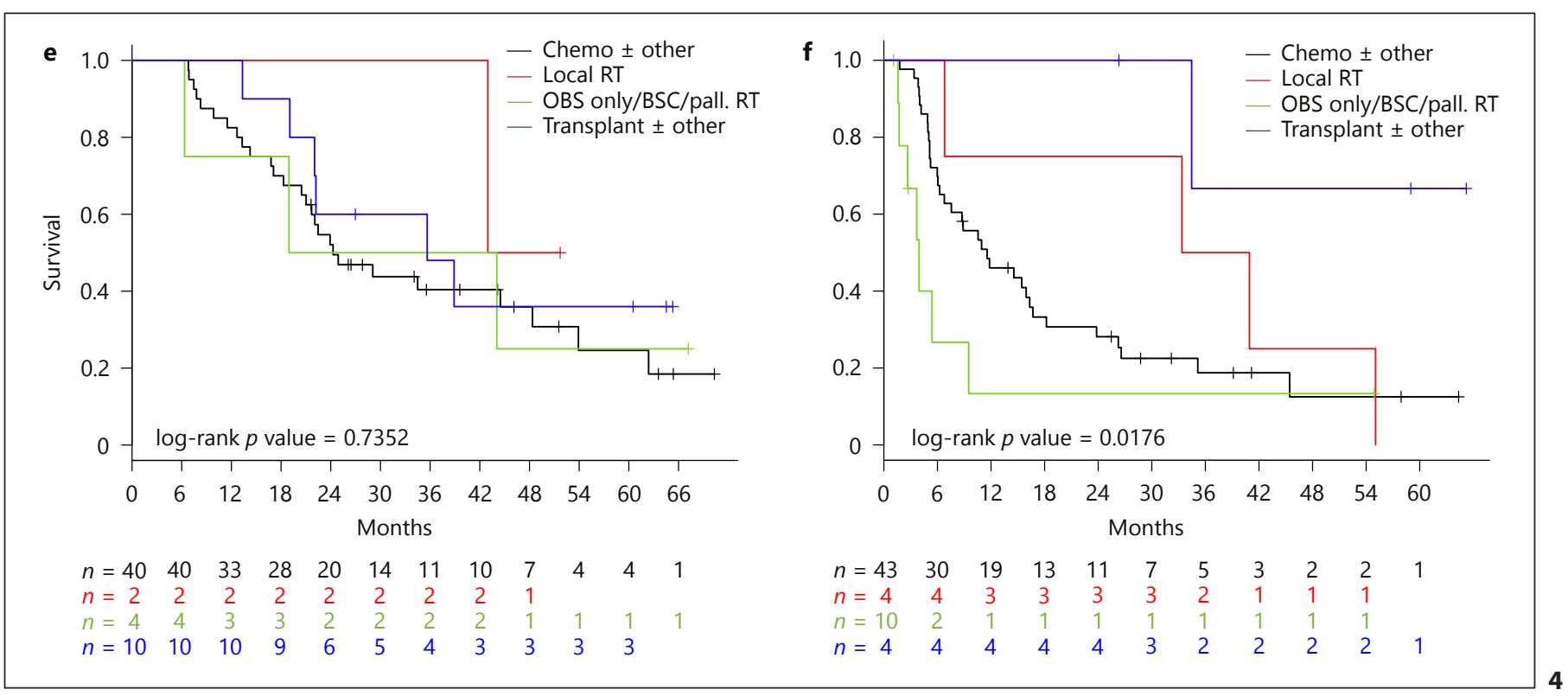

at diagnosis with a more global demographic representation than COMPLETE. In this study, a similar percentage of patients (436 patients of 1,020$)$ were reported to have relapsed or refractory disease [12]. The median OS reported by the T-Cell Project was 5.8 months compared to 20.5 months in our study. The different outcomes between these studies may be explained in part by the broader distribution of patients across multiple international sites, different numbers of patients with rare and less favorable subtypes, and differences in availability and use of novel agents and stem cell transplant in the salvage setting among the different countries represented.

Another difference between these registry studies is that COMPLETE captured information regarding physician intent for palliative or potentially curative treatment for refractory and relapsed patients. We found that age and not histology or other patient-related factors was the predominant factor dictating treatment goals. Also, the availability of multiple novel FDA-approved agents in the United States at the time that our study was conducted allowed us to identify different patterns of use of single agent versus multi-agent approaches, with more use of single agents in refractory versus relapsed patients. A future merge of data from these two registries and others could provide larger numbers of patients to further define factors predictive of refractory or relapse disease and outcomes with specific therapeutic agents or regimens in the refractory and relapsed setting.

Given that our study was observational, our results may be biased based on a number of patient- and center- specific factors; nevertheless, our study, like the T-Cell Project, represents the "real-world" landscape of treatment regimens and outcomes for patients with newly diagnosed PTCL in both academic and community settings and defines for the first time in a prospective design the treatment patterns for patients with these rare diseases.

In summary, our study along with data from the T-Cell Project demonstrate that outcomes for patients with PTCL who do not achieve a CR to front-line therapy remain poor, with only a small proportion of those patients achieving a CR in the relapsed or refractory setting, and a smaller fraction undergoing a potentially curative stem cell transplant. In the era of targeted and pathway-directed therapies, the role of these novel agents in the treatment algorithm for aggressive T cell lymphomas has yet to be defined. Our patient cohort demonstrates that early utilization of single agents over combination chemotherapy has been mostly in patients with relapsed disease and that single agents can serve as a bridge to potentially curative stem cell transplants. Our study highlights the need for prospective databases like COMPLETE to further redefine current treatment practices for patients with refractory and relapsed PTCL and support the concept that whenever possible these patients should be enrolled in clinical trials.

\section{Statement of Ethics}

Subjects have given their written informed consent. The study protocol has been approved by the research institute's committee on human research. 


\section{Disclosure Statement}

The authors have no relevant conflicts of interest to declare.

\section{Funding Sources}

This work was supported by Spectrum Pharmaceuticals Inc.

\section{Author Contributions}

All the authors listed meet ICMJE criteria for authorship in that they have all: made substantial contributions to the conception or design of the work, or the acquisition, analysis, or interpretation of data for the work; and they have participated in drafting the work or revising it critically for important intellectual content; and they have given final approval of the version to be published; and they have agreed to be accountable for all aspects of the work in ensuring that questions related to the accuracy or integrity of any part of the work are appropriately investigated and resolved.

\section{References}

1 Moskowitz AJ, Lunning MA, Horwitz SM. How I treat the peripheral T-cell lymphomas. Blood. 2014 Apr;123(17):2636-44.

2 Coiffier B, Federico M, Caballero D, Dearden $\mathrm{C}$, Morschhauser F, Jäger U, et al. Therapeutic options in relapsed or refractory peripheral $\mathrm{T}$ cell lymphoma. Cancer Treat Rev. 2014 Oct; 40(9):1080-8.

3 Vose J, Armitage J, Weisenburger D; International T-Cell Lymphoma Project. International peripheral T-cell and natural killer/Tcell lymphoma study: pathology findings and clinical outcomes. J Clin Oncol. 2008 Sep; 26(25):4124-30.

4 Mak V, Hamm J, Chhanabhai M, Shenkier T, Klasa R, Sehn LH, et al. Survival of patients with peripheral T-cell lymphoma after first relapse or progression: spectrum of disease and rare long-term survivors. J Clin Oncol. 2013 Jun;31(16):1970-6.

5 Zinzani PL, Bonthapally V, Huebner D, Lutes R, Chi A, Pileri S. Panoptic clinical review of the current and future treatment of relapsed/ refractory T-cell lymphomas: Peripheral Tcell lymphomas. Crit Rev Oncol Hematol. 2016 Mar;99:214-27.
6 Jacobsen E, Freedman AS. Treatment of relapsed or refractory peripheral T cell lymphoma. In Basow DS, editor. UpToDate. Alphen aan den Rijn: Wolters Kluwer, 2016.

7 National Comprehensive Cancer Network. Non-Hodgkin lymphomas. Version 2.2016 [Accessed 2016, Nov 6]. Available from http://www.nccn.org/professionals/physician_gls/pdf/nhl.pdf.

8 Lansigan F, Seropian S, Cooper D, Foss F. A retrospective comparison of autologous vs. allogeneic transplantation for peripheral T-cell lymphoma: a single institution experience. Blood. 2008;112(11):4392.

9 Horwitz S, Moskowitz C, Kewalramani T, Hamlin P, Straus D, O'Connor O, et al: Second-line therapy with ICE followed by high dose therapy and autologous stem cell transplantation for relapsed/refractory peripheral T-cell lymphomas: Minimal benefit when analyzed by intent to treat. Blood. 2005;106: 2679.

10 Smith SD, Bolwell BJ, Rybicki LA, Brown S, Dean R, Kalaycio M, et al. Autologous hematopoietic stem cell transplantation in peripheral T-cell lymphoma using a uniform high- dose regimen. Bone Marrow Transplant. 2007 Aug;40(3):239-43.

11 Tarella C, Gueli A, Delaini F, Rossi A, Barbui AM, Gritti G, et al. Rate of primary refractory disease in B and T-cell non-Hodgkin's lymphoma: correlation with long-term survival. PLoS One. 2014 Sep;9(9):e106745.

12 Bellei M, Foss FM, Shustov AR, Horwitz SM, Marcheselli L, Kim WS, et al.; International T-cell Project Network. The outcome of peripheral T-cell lymphoma patients failing first-line therapy: a report from the prospective, International T-Cell Project. Haematologica. 2018 Jul;103(7): 1191-7.

13 d'Amore F, Relander T, Lauritzsen GF, Jantunen E, Hagberg $\mathrm{H}$, Anderson $\mathrm{H}$, et al. Up-front autologous stem-cell transplantation in peripheral T-cell lymphoma: NLG-T-01. J Clin Oncol. 2012 Sep;30(25): 3093-9.

14 Carson KR, Horwitz SM, Pinter-Brown LC, Rosen ST, Pro B, Hsi ED, et al. A prospective cohort study of patients with peripheral T-cell lymphoma in the United States. Cancer. 2017 Apr;123(7):1174-83. 